\section{The politics of fear}

\section{What right-wing populist discourses mean}

\author{
Ruth Wodak \\ London - Los Angeles 2015: Sage, 256 Seiten.
}

Matthias Falter

Institut für Zeitgeschichte, Universität Wien, Österreich

E-Mail: matthias.falter@univie.ac.at

Angst ist als Topos und Mittel der Politik allgegenwärtig. Die Konstruktion von Bedrohungsszenarien zur Durchsetzung politischer Ziele und die Aufforderung, die Ängste der BürgerInnen ernstzunehmen, sind dabei oft nur unterschiedliche Seiten der gleichen Medaille. Vor allem extrem rechte und populistische Parteien profitieren von Politiken der Angst in Europa und darüber hinaus. In ihrem neuesten Buch untersucht Ruth Wodak diese „micro-politics of right-wing populist parties - how they actually produce and reproduce their ideologies and exclusionary agenda in everyday politics, in the media, in campaigning, in posters, slogans and speeches" (2).

Ihre Erfolge seien, so Ruth Wodak, nicht zuletzt auch ein Resultat der „normalization of nationalistic, xenophobic, racist and antisemitic rhetoric“ $(\mathrm{x})$. Verstärkt und legitimiert werde diese Normalisierung durch die Übernahme rechtspopulistischer Terminologie und Bilder durch Parteien in der politischen „Mitte“. Trotz aller regionalen und nationalen Unterschiede extrem rechter Parteien ist die Produktion von Angst gewissermaßen ein gemeinsamer Nenner - ein zentrales diskursives Mittel, das nicht zuletzt dazu dient, Politiken der Exklusion und Ungleichheit zu legitimieren.

Während in der Politikwissenschaft unter dem Begriff des „(Rechts-)Populismus“ häufig ein spezifischer "Politikstil“ verstanden wird, betont Wodak, dass auch die Inhalte rechtspopulistischer Politik mehr in den Blick genommen werden sollten. Ausgehend von einer konzeptionellen Annäherung an das Phänomen Rechtspopulismus richtet Wodak den Blick auf die einzelnen Aspekte von Politiken der Angst. „Politics of identity" haben zur Entstehung neuer Konfliktlinien abseits von „links“ und „rechts“ beigetragen. Durch eine
Renationalisierung und die Ethnisierung von sozialen Konflikten werden Bedrohungsbilder erzeugt, die nicht zuletzt eine antagonistische Politik des „Wir“ gegen das „Sie“ legitimieren sollen.

Die Studie verbindet in erhellender Weise sprachund politikwissenschaftliche Aspekte. Besonders die ausführlich kontextualisierten Beispiele rassistischer, antisemitischer und sexistischer Äußerungen und Inszenierungen von extrem rechten PolitikerInnen machen den Band sowohl zu einer instruktiven Einführung als auch interessanten und notwendigen Ergänzung für den Forschungsgegenstand Rechtspopulismus. Die Beispiele reichen dabei von Heinz-Christian Straches Facebook-Posting einer antisemitischen Karikatur über die Konstruktion eines „christlichen“ oder „jüdischchristlichen" Europas bis hin zu Debatten über ,den Islam'. Dabei werden auch die Karrieren von Motiven über europäische Grenzen hinweg beleuchtet, z.B. das Plakat der rassistischen "Ausschaffungsinitiative“ in der Schweiz 2007, das sowohl von der deutschen NPD als auch von der italienischen Lega Nord übernommen wurde. Ein Glossar am Ende des Buches mit Informationen zu rechtsextremen und rechtspopulistischen Parteien in Europa sowie zur Tea Party in den USA macht das Buch zusätzlich auch als Nachschlagewerk wertvoll. Kleinere Detailfehler sollten in der wünschenswerten nächsten Auflage jedoch korrigiert werden. So hat Andreas Mölzer nach dem erzwungenen Rücktritt von der Kandidatur 2014 nicht die FPÖ verlassen, und Strache ist kein Mitglied der Burschenschaft Olympia.

Besonders erfreulich ist, dass Wodak einen Aspekt beleuchtet, der sowohl im medialen Diskurs als auch in der wissenschaftlichen Auseinandersetzung mit dem Phänomen selten thematisiert wird: die Frage des Ge- 
schlechterverhältnisses und der Geschlechterpolitiken der extremen Rechten. Während weiterhin patriarchale Ideologien des Regierens und Regulierens weiblicher Körper, zentraler Bestandteil rechtspopulistischer und rechtsextremer Politiken sind, werden gleichzeitig Fragmente feministischer Diskurse angeeignet, umgedeutet, und gegen die „Anderen“ insbesondere gegen Muslime gewendet.

Politiken der Angst sind jedoch nicht alternativlos, sondern erfordern sinnvolle Gegenpositionen. Wodak schließt mit Vorschlägen einer Politik, die rechtspopulistischen Argumenten entgegenwirkt, und fordert inkludierende Ansätze, die Dichotomien aufbrechen. „Not falling into the trap would thus entail a politics of solidarity instead of a politics of fear or envy“. (I89) 